\title{
Theoretical Calculation of Laser Driving 3-D Microrotors
}

\author{
Liu Yuxiang, Zhu Anding, Huang Wenhao \\ Department of Precision Machinery and Precision Instrumentation, Univ. of Science and Technol- \\ ogy of China, Hefei, Anhui, 230026, P.R.China \\ E-mail: whuang@ustc.edu.cn
}

\begin{abstract}
In this paper, we propose the new theoretical investigation on the optical forces and torques on complex microrotors. Based on R. C. Gauthier's modified ray model, the optical forces and toques on a complex micro-object, the conical microrotor, are analyzed. The viscous drag torque is estimated by Stokes flow to obtain the rotational speed. The results of our computation agree well with the previously published experimental results, which indicates that our approach of the optical torque calculation is suitable for complex microrotors and that the theoretical calculation is very helpful to optimum design of light-driven microrotors. The primary experimental results of one kind of microrotors driven are also presented.
\end{abstract}

DOI: $10.2961 /$ jlmn.2006.02.0007

Keywords: optical tweezers, microrotors, spin symmetry, gradient force

\section{Introduction}

The techniques of optical trapping and manipulation of micro-objects are getting increasing attention in the MEMS field and biological applications. "Optical tweezers" is the common term to describe the radiation pressure induced optical force generation by a highly convergent laser beam. It has been experimentally demonstrated that particles can be trapped, manipulated, oriented and spun in these laser beams [1-5]. Knowledge of the traping forces in terms of their magnitudes and directions is important to understand and predict the interaction of the focused laser beam with various micromachines and cells. In previous work, two main models have been used to analyze the optical trapping force: the ray optics model for $\mathrm{R}>5 \lambda$, and the electromagnetic model for $\mathrm{R}<\lambda / 2$, where $\mathrm{R}$ is the feature size of the particles and $\lambda$ is the wavelength. In 1995, a hybrid ray-wave model was presented by R. C. Gauthier, which could be used for the particles with the feature size of $\mathrm{R} \approx \lambda[6]$. Later the same author presented the term "point ray" to improve rationality of the model [7]. Gauthier discussed the behaviors of many micro-objects in the laser beams with this model, such as spheres, rings, cylinders and cubes [8]. However, the theoretical investigations have been focused on basic geometries such as spheres, near-spheres, etc. This is due to the difficulty to fabricate complex particles and that many biological particles can be approximate with spheres. In the work that has been done on the rotation of microrotors, the microrotors were simple-shaped, such as the cross-shaped microrotor [6] and the sloping microrotor [9].

In 2001 and 2002, Péter Galajda produced and rotated some complex microrotors experimentally, which promised great practical potential in MEMS and nanotechnology applications. But there has been no related theoretical investigation about these complex microrotors as far as our concern. In this paper, Gauthier's modified ray model is used as the theoretical foundation to study one complex microrotor rotated in laser tweezers: the conical microrotor.
The optical forces and toques on the microrotors are calculated, and their rotational speeds are obtained by estimating the viscous drag torque, which are in good agreement with Galajda's experimental results.

\section{Methodology}

The theoretical expressions required for calculating the forces and the torques exerted on the microrotors are derived with the hybrid ray-wave optical model, which combines Gaussian mode profile with the ray optical model [7]. The incident direction and the incident light intensity are determined by the Guassian mode beam. For a $\mathrm{z}$ axis directed laser beam with the least waist at $\mathrm{z}=0$, the incident direction of an arbitrarily specified point $\mathrm{P}\left(\mathrm{x}_{\mathrm{t}}, \mathrm{y}_{\mathrm{t}}, \mathrm{z}_{\mathrm{t}}\right)$ on the microrotor surface is perpendicular to the wavefront at the point P. Fig. 1 shows the geometrical relationship for the incident photon direction calculation. $\mathrm{C}$ is the center of the spherical wavefront at the point $\mathrm{P}$, then the radius $\mathrm{R}$ and $\mathrm{dz}$ can be obtained by solving the equations set:

$$
\left\{\begin{array}{l}
R=\left(z_{t}+d z\right) \times\left(1+\frac{z_{0}}{z_{t}+d z}\right) \\
R^{2}=(R-d z)^{2}+x_{t}^{2}+y_{t}^{2}
\end{array} \text { where } z_{0}=\frac{\pi \omega_{0}^{2}}{\lambda}\right.
$$

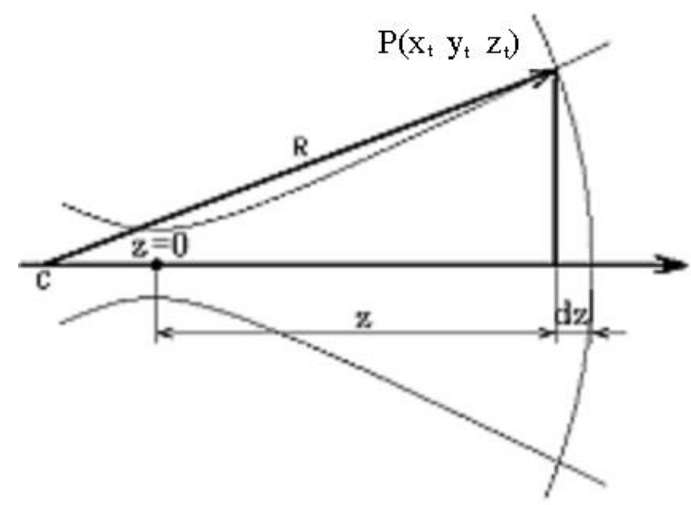

Fig. 1 The geometrical relationship for the incident beam pencil direction calculation 
Here, $\omega_{0}$ is the least beam waist, $\lambda$ the vacuum wavelength. Then the incident direction can be expressed as

$$
\overrightarrow{i n}=\overrightarrow{C P}\left(x_{t}, y_{t}, R-d z\right)
$$

The incident light intensity I $(x, y, z)$ at $P\left(x_{t}, y_{t}, z_{t}\right)$ is

$$
I\left(x_{t}, y_{t}, z_{t}\right)=\frac{2 P}{\pi \omega_{z}^{2}} \exp \left[\frac{-2\left(x_{t}^{2}+y_{t}^{2}\right)}{\omega_{z}^{2}}\right]
$$

Here, $\mathrm{P}$ is the total laser power, $\omega_{z}$ the beam waist on the plane $z=z_{t}$ and can be expressed as

$$
\omega_{z}=\omega_{0} \sqrt{1+\left(z_{t} / z_{0}\right)^{2}}
$$

For a small region $\mathrm{dS}$ at the point $\mathrm{P}(\mathrm{x}, \mathrm{y}, \mathrm{z})$ illuminated by laser beam, during the time interval $\mathrm{dt}$, $\mathrm{Nt}$ being the number of incident photons is expressed as

$$
N_{t}=\frac{I(x, y, z)}{h c}(-\overrightarrow{i n} \cdot \overrightarrow{n o r}) \lambda d t d S
$$

Here $\mathrm{h}$ is the Planck constant, c the vacuum light speed. The factor $(-\overrightarrow{i n} \cdot \overrightarrow{n o r})$ means that the light intensity should be projected to the interface normal direction in order to gain the incident energy. It is assumed that the normal vector of the region $\mathrm{dS}$, the incident direction vector, the reflect direction vector, the refract direction vector are $\overrightarrow{n o r}, \overrightarrow{i n}, \overrightarrow{\text { reflect }}, \overrightarrow{\text { refract }}$ respectively, and that the refractive indexes of the incident medium and the refractive medium are $\mathrm{n} 1, \mathrm{n} 2$. The momentum transferred to the surface from one photon is expressed as

$$
\overrightarrow{d P_{0}}=\frac{h}{\lambda}\left(n_{1} \overrightarrow{i n}-n_{1} R \cdot \overrightarrow{\text { reflect }}-n_{2} T \cdot \overrightarrow{\text { refract }}\right)
$$

Here, $\mathrm{R}$ and $\mathrm{T}$ are the Fresnel reflection and refraction coefficients. If the beam is unpolarized, they are expressed as

$$
R=\frac{1}{2}\left[\frac{\tan ^{2}\left(\theta-\theta^{\prime}\right)}{\tan ^{2}\left(\theta+\theta^{\prime}\right)}+\frac{\sin ^{2}\left(\theta-\theta^{\prime}\right)}{\sin ^{2}\left(\theta+\theta^{\prime}\right)}\right], T=1-R
$$

Here, $\theta$ is the incident angle, $\theta^{\prime}$ the refraction angle. Then the optical force exerted on the small region can be obtained as

$$
\begin{aligned}
& \overrightarrow{d F}=\frac{\overrightarrow{d P}}{d t}=\frac{N_{t} \overrightarrow{d P_{0}}}{d t} \\
& =\frac{I(x, y, z)}{c} \times\left(n_{1} \overrightarrow{i n}-n_{1} R \cdot \overrightarrow{\text { reflect }}-n_{2} T \cdot \overrightarrow{\text { refract }}\right)(-\overrightarrow{i n} \cdot \overrightarrow{\text { nor }}) d S
\end{aligned}
$$

The vector integral of all the forces elements for each illuminated small surface region dS presents the total forces exerted by the laser beam on the microrotors. And the torque element integral presents the total torque exerted by the laser beam, which is most interesting in this paper:

$$
\vec{F}=\int_{\text {allregion }} \overrightarrow{d F}, \quad \vec{M}=\int_{\text {allregion }} \overrightarrow{d M}=\int_{\text {allregion }} \vec{r} \times \overrightarrow{d F}
$$

\subsection{Calculation of the optical torque and rotational speed of the conical microrotor}

Now we consider the optical torque of the conical microrotor that is rotated in solution (shown in Fig. 2. a.). It is obvious that the eight legs of the microrotor contain a spin symmetry about a central axis, which means that the total toque is 8 times of the toque exerted on one leg. So we construct the coordinate system (shown in Fig. 2. b.) to calculate the toque on one leg. The xyz coordinate system is the world coordinate system, and the x'y'z' coordinate system is the computational coordinate system. Because the microrotor is symmetric, the beam axis coincides with the microrotor axis when it gets the stable state. In our model, the Gaussian laser beam axis coincides with the rotor axis, and the beam transmits along the negative $\mathrm{z}$ coordinate. The y' axis coincides with the cylinder axis of the leg, the $z$ axis parallels the laser beam axis, the $\mathrm{x}$ axis point to the beam axis and coincides with the $x$ ' axis. The inclination angle of the rotor legs is $45^{\circ}$, so when the xyz coordinate system rotates $45^{\circ}$ clockwise about the $\mathrm{x}$ axis, it coincides with the x'y'z' coordinate system. a)

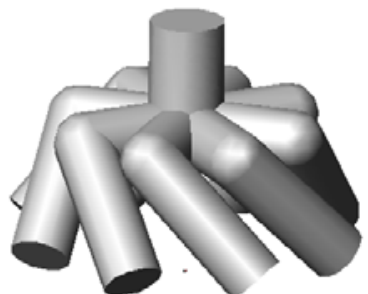

1)

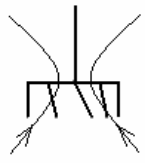

2)

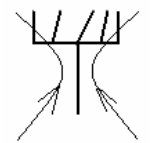

b)

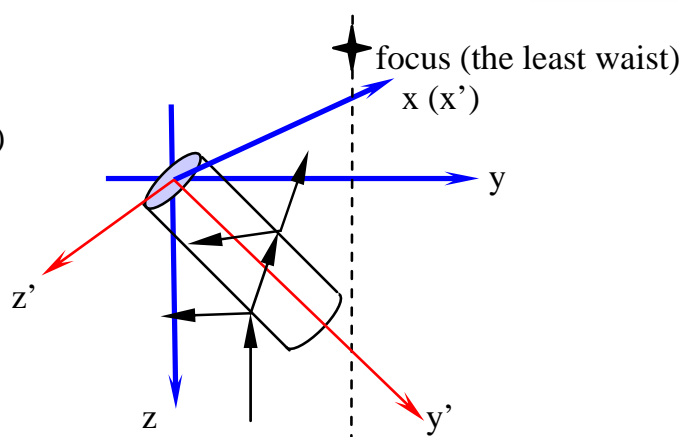

Fig. 2 a). The conical rotor to calculate; b). the coordinate system used in the calculation

As discussed above, the toque is obtained by the integral of the toques on each small surface region. In our model, the integral is performed in two dimensions: one is the length y' along the cylinder axis and the other is the angle $\theta$ in the direction of the tangent to the cylinder, so the first thing is to determine the illuminated angle range for a fixed y'. We take the following steps. First we find the central incident beam pencil of the illuminated annular region for a fixed y' by finding the incident beam pencil at $\left(0, y^{\prime}, 0\right)$ in the $x^{\prime} y^{\prime} z^{\prime}$ coordinate system, and this beam pencil intersects the cylinder surface at $\left(r \cos \theta_{0}, y^{\prime}, r \sin \theta_{0}\right)$. Here, $\mathrm{r}$ is the cylinder radius. Then each time a pace is subtracted from (or added to) the incident angle $\theta_{0}$, until the incident direction vector is perpendicular to the cylinder surface normal vector at the point $\left(r \cos \theta, y^{\prime}, r \sin \theta\right)$. In this way the illuminated annular region for a fixed $y^{\prime}$ is determined. Every step we can get one small region dS at the point $\left(r \cos \theta, y^{\prime}, r \sin \theta\right)$, and the intensity and direction of the incident light beam pencil at this point is calculated after converting the point coordinate to xyz coordinate system by multiplying a coordinate conversion matrix: 


$$
T=\left[\begin{array}{ccc}
1 & 0 & 0 \\
0 & \cos \frac{\pi}{4} & \sin \frac{\pi}{4} \\
0 & -\sin \frac{\pi}{4} & \cos \frac{\pi}{4}
\end{array}\right]
$$

Then the force and the torque of the first-time incident are calculated. The refracted beam travels in the rotor body and makes the second-time, the third-time...incidence, and each incidence contributes to the torque on the microrotor. There are two possibilities for these incidences because of the finitude of the cylinder length: incidence at the cylinder surface or incidence at the end surface. The force and torque of each incidence are calculated and added to the total torque for the region dS. We calculate five incidences for one beam pencil incident to dS using ray tracing. There are two main reasons for calculating only five incidences: 1 ) the error for five incidences and ten incidences is only about 1 percent; 2) some of the light intensity is absorbed when traveling in the rotor body. The total toque is obtained by summing the torques of all illuminated small regions.

When a particle has a feature size of 0.1 10 micron, its movement in fluid medium is the application of low Reynolds number hydrodynamics, which is also called "Microhydrodynamics" [10]. In such a dimension, the inertial effect of the fluid is neglected, and the flow field can be approximated by Stokes flow. Considering that the results of Stokes flow are insensitive to particle shape, reasonable result is obtained by approximating the viscous drag torque with a sphere. Furthermore, according to Galajda's work [4], the viscous drag torque of the conical microrotor is approximately equal to a sphere of the same diameter at the rotation speed of $10 \mathrm{~s}^{-1}$, and the linear correlation between rotational speed and drag torque is a good approximation in a wide range of velocities. So the viscous drag torque of the conical microrotor is approximated by a rotating sphere of the same radius

$$
D=-8 \pi \mu \omega S r^{3}
$$

Here $\mu$ is the constant viscosity coefficient, $\omega$ the rotational speed. When the microrotor gets the stable state, the optical torque is equal to the viscous drag torque, and the rotational speed is obtained.

\section{Results and Discussion}

In our calculation, all the parameters are chosen referring to Péter Galajda's experiment for a compare with the experimental results [4]. The parameters used are as follows: the refractive index of the solution is the solution of the acetone $n_{1}=1.36$, the refractive index of the microrotor $n_{2}=1.56$, the wavelength of the laser $\lambda=0.994 \mu \mathrm{m}$, the microrotor radius $S r=2 \mu \mathrm{m}$, the length of the leg $h=2.5 \mu \mathrm{m}$, the radius of the leg cylinder $r=0.35 \mu \mathrm{m}$, the distance of the laser focus from the top end face of the microrotor lfocus $=-5 \mu \mathrm{m}$ (the negative sign indicates that this length is measured along the negative $\mathrm{z}$ coordinate direction), and the inclination angle of the conical rotor's leg $\alpha=45^{\circ}$.

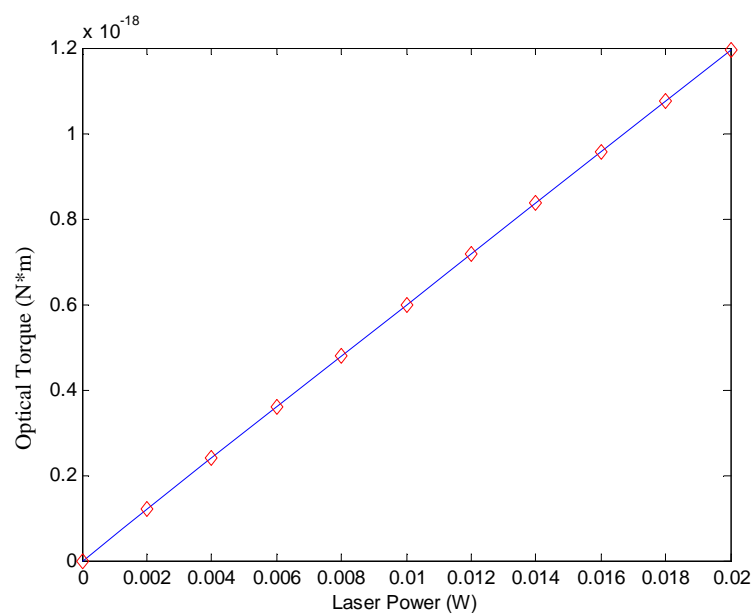

Fig. 3 Dependence of the optical torque upon the laser intensity

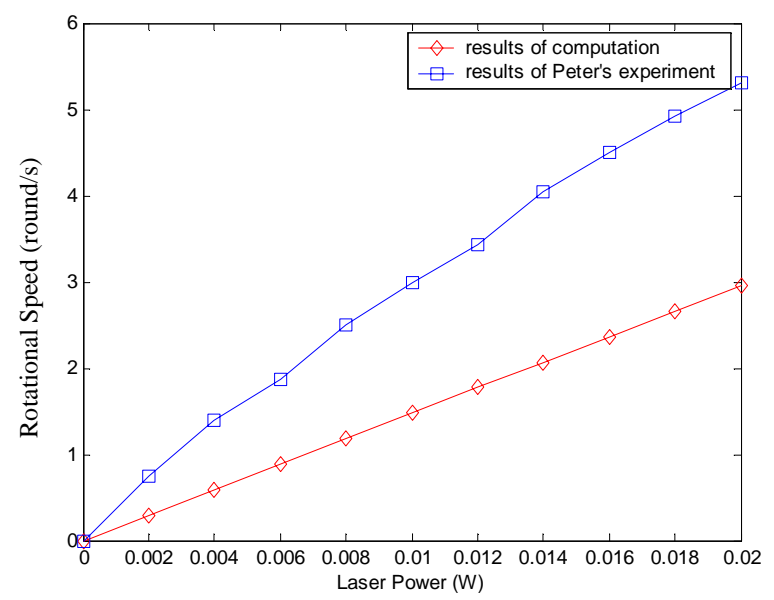

Fig. 4 Dependence of the rotational speed upon the laser intensity and the compare of our computational results and Peter's experimental results

The plots of the optical torque and the rotational speed as a function of the laser power are shown in Fig. 3 and Fig. 4. The optical torque exerted on the microrotor is of the order of magnitude of $10^{-18} \mathrm{~N} \cdot \mathrm{m}$ and is linearly dependent on the laser power. Also, the rotational direction is the same with the experiment. Fig. 4 presents that our computational rotational speed is in good agreement with the experimental results. Though the computational results is about half of the experimental results, the gap is acceptable in such a small dimension. The reason for the gap may be the error of estimating the microrotor's viscous drag torque by a sphere. The drag torque of a sphere of the same radius is larger than that of the microrotor. When a Gaussian mode laser beam with no spin symmetry travels by the inclined legs with a spin symmetry, the emergent beam will contain a spin symmetry, too. Then the emergent beam will exert extra torques on the protruding structures of the microrotor that coincides with the $\mathrm{x}$ axis shown in Fig.2. $\mathrm{b}$, and these extra torque also contributes to the deviation between the computational results and the experimental results. It must be clarified that it is mentioned by Péter Gala- 
jda that there are two stable positions the rotor can assume in the laser trap (shown in Fig.3) [4]. In the second position shown as Fig.3, the beam is incident to the haft and scattered before the beam arrive at the legs, so the torque of this position is smaller than the first position and hard to calculate. In this consideration, all our results are for the first position.

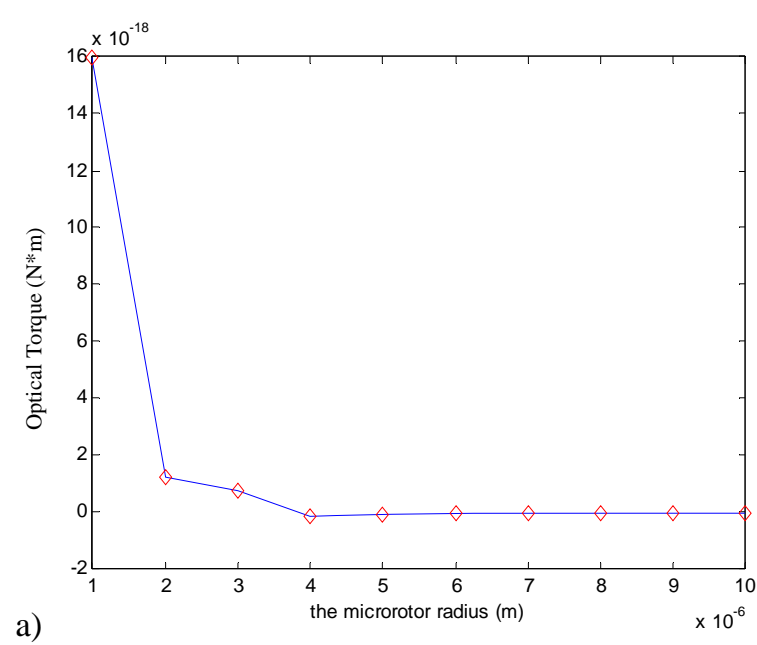

a)

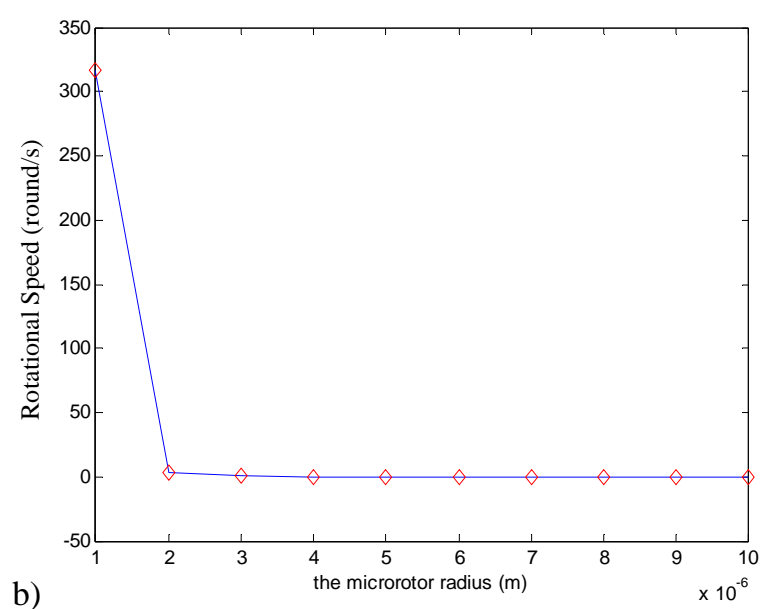

Fig. 5. a). Dependence of the optical torque upon the microrotor radius; b). Dependence of the rotational speed upon the microrotor radius

Dependences of the optical torque and the rotational speed upon the microrotor radius are shown as Fig. 5. When the radius of the microrotor increases in the computation, the length of the leg also increases proportionally. It is obvious that microrotors with smaller size increase their rotational speed largely. Dependence of the optical torque upon the laser focus position is shown in Fig. 6. It shows that when the laser focus is close to the legs, the torque is small. This is because the distribution of the laser intensity on a plane perpendicular to the laser beam axis is sharp when the plane is close to the focus plane. When the laser focus is close to the microrotor, the laser intensity incident to the legs is small. The intensity grows higher as the focus leaves the microrotor, so the optical torques grows larger. When the focus is too far away from the microrotor, the laser power disperses in a large area, so the intensity grows smaller. In this case, the optical torque increases slower and decreases finally. Furthermore, when the focus is too far away from the microrotor, it can't assume the stable position. Dependence of the optical torque upon the inclination angle of the conical rotor's leg is shown in Fig. 7, which indicates that the inclination angle of $45^{\circ}$ is very efficient for the microrotor rotation and may be the best choice of the inclination angle.

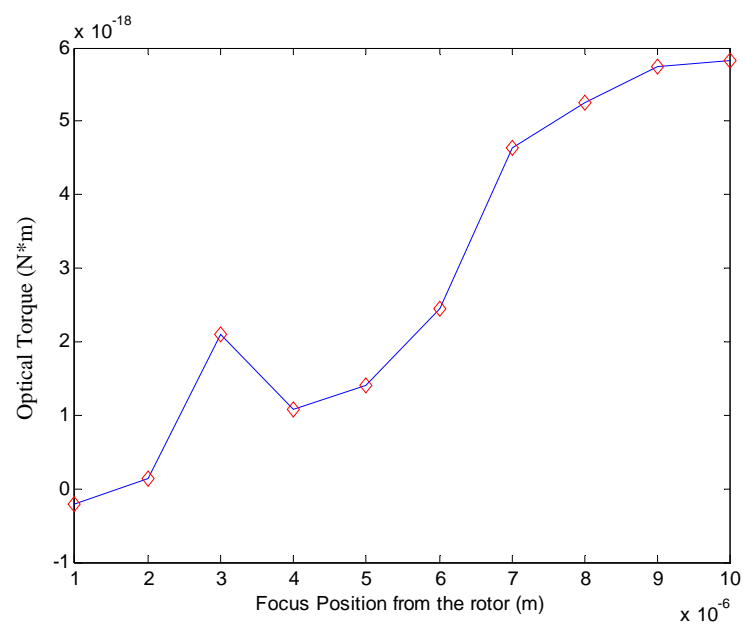

Fig. 6. Dependence of the optical torque upon the laser focus position

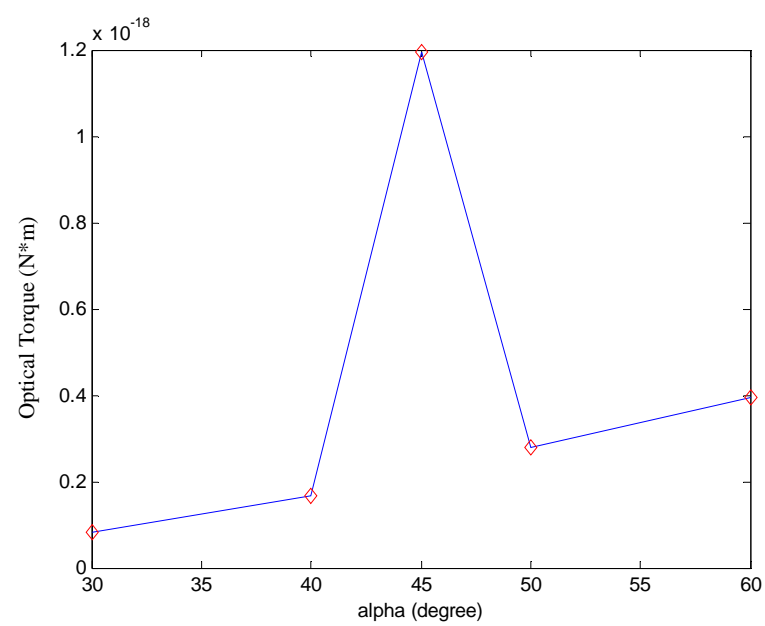

Fig. 7. Dependence of the optical torque upon the inclination angle of the conical rotor's leg

\section{Experimental Results}

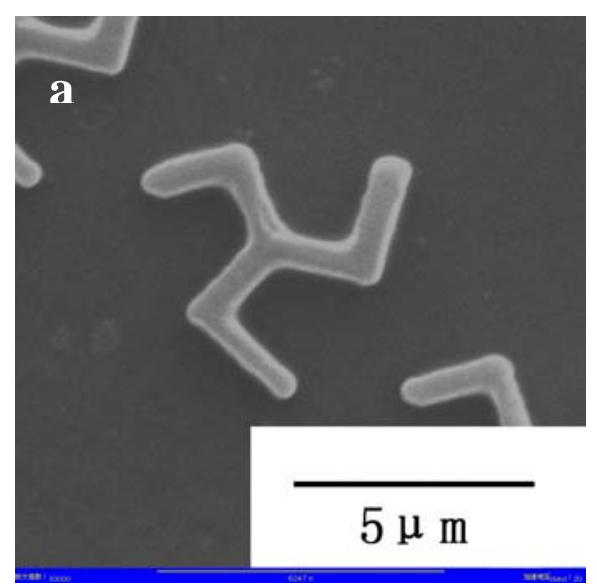



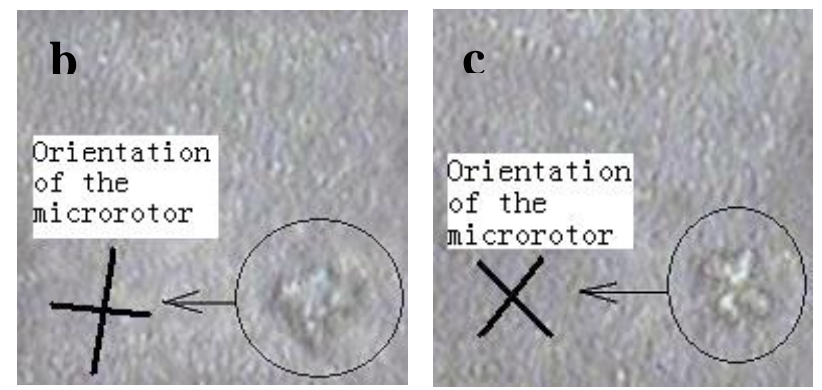

Fig. 8 The microscopy photographs of the microrotors. a) is the SEM image of the microrotor with three legs. (b) and (c) are the microrotor with four legs during rotating with two different orientations. The diameter of the microrotor is about $5 \mathrm{um}$. (b) and (c) are taken from the video of the optical microscope.

We have fabricated two kinds of microrotors with three and four legs by femtosecond laser two-photon fabrication method, and the microrotor with four legs is successfully rotated with laser tweezers. The diameter of the microrotor is about 5um. The rotation speed can be about $200 \mathrm{rpm}$ when the laser power is $50 \mathrm{~mW}$ before the objective. The SEM image of the microrotor with three legs is shown as Fig. 8 (a), and the rotated microrotor with four legs is shown as Fig. 8 (b). Further experiments are still in progress.

\section{Conclusion}

We have proposed the suitable and efficient theoretical approach to calculate the optical torque of complex microrotors based on the modified ray model. Our computational results agree well with Péter Galajda's experiment results, and this indicates that this model is appropriate for the optical forces and torques computation of the microrotors with a feature size comparable to the light wavelength. The dependences of the optical torques upon some parameters of the laser and the microrotor are analyzed. The approach is general to cylindrical structures and can be easily applied to other micro-objects with cylindrical structures, especially to other complex microrotors. The simulation is very helpful to the optimization of the microrotor design. Two kinds of microrotors with three and four legs are fabricated by femtosecond laser two-photon fabrication method, and the microrotor with four legs is successfully rotated with laser tweezers. Additionally, the theoretical investigation of the optical forces on other complex microrotors and of other exciting phenomena related to optical forces will be pursued by the authors.

\section{Acknowledgments}

The work was supported by National Nature Science Foundation of China (No.50275140 and No.50335050).

\section{References}

[1] Ashkin, A., J. M. Dziedzic, J. E. Bjorkholm and S. Chu: Optics Lett., 11, p. 288-290, 1986. (Journals)

[2] Péter Galajda and Pál Ormos: Optics Express, 11, p. 446-451, 2003. (Journals)

[3] Hiroo Ukita and Motoki Kanehira: IEEE Journal on Selected Topics in Quantum Electronics, 8, p. 111-117, 2003. (Journals)
[4] Péter Galajda and Pál Ormos: Applied Physics Letters, 78, p. 249-251, 2000. (Journals)

[5] Péter Galajda and Pál Ormos: J. Opt. B: Quantum Semiclass. Opt., 4, p. S78-S81, 2002. (Journals)

[6] R. C. Gauthier: Appl. Phys. Lett., 67, p. 2269-2271, 1995. (Journals)

[7] R. C. Gauthier: Optics \& Laser Technology, 29, p. 389-399, 1997. (Journals)

[8] R. C. Gauthier and Athanasios Frangioudakis: Applied optics, 39, p. 3060-3070, 2000. (Journals)

[9] Hiroo Ukita and Kenji Nagatomi: Applied Optics, 42, p. 2708-2715, 2003. (Journals)

[10] Yan Zongyi: "Low Reynolds Number Flow Theory”, (publisher: Beijing University Press), 2002. (Books)

(Received: April 4, 2005, Accepted: March 27, 2006) 